\title{
Malignant Fibrous Histiocytoma with In-Transit Metastasis
}

\author{
Taku Fujimura ${ }^{a}$ Masayuki Sugawara ${ }^{a}$ Takahiro Haga ${ }^{a}$ \\ Yoshiyuki Kariya $^{b}$ Ryuhei Okuyama ${ }^{a}$ Hachiro Tagami ${ }^{a}$ \\ Setsuya Aiba ${ }^{a}$
}

Departments of a Dermatology and ${ }^{\mathrm{b}}$ Pathology, Tohoku University Graduate

School of Medicine, Sendai, Japan

\section{Key Words}

Malignant fibrous histiocytoma $\cdot$ CD99 $\cdot$ In-transit metastasis · Atypical fibroxanthoma

\begin{abstract}
Malignant fibrous histiocytoma (MFH) is the most common fibroblastic tumor, but its cutaneous metastasis, especially in-transit metastasis, is extremely rare. We describe the case of a 30-year-old Japanese man with a recurrent MFH on the scalp accompanied by in-transit metastasis, which had been treated as a benign skin tumor 8 years before. The main bulk of the recurrent tumor was located in the dermis, but the metastatic tumor was mainly located in the subcutis. Generally, atypical fibroxanthoma, also known as cutaneous MFH, is rarely metastasized and presents a benign clinical course. Since there is a great difference between the prognosis of MFH and atypical fibroxanthoma, precise diagnosis of the primary tumor is essential.
\end{abstract}

\section{Introduction}

Malignant fibrous histiocytoma (MFH) is the most common soft tissue sarcoma of late adult life, and it is rare in young patients. It usually involves the deep soft tissues and the striated muscles of the proximal part of the extremities, particularly the lower extremities. Up to $25 \%$ of cases may arise in subcutaneous tissues, although less than $10 \%$ are confined to the subcutis without underlying fascial involvement [1]. The superficial form of cutaneous MFH is known as atypical fibroxanthoma (AFX). Although AFX is histologically indistinguishable from MFH, AFX invariably shows a more favorable prognosis that results from its superficial location [1]. More recently, O13 (CD99) was found to display immunoreactivity in cases of AFX, but it was negative for MFH [2].

CD99, also known as p30/32 glycoprotein or MIC-2 protein, is a cell surface protein encoded by genes on the $\mathrm{X}$ and $\mathrm{Y}$ chromosomes. It has been shown to be reactive in 
Ewing's sarcoma, primitive neuroectodermal tumors, some lymphomas, and other mesenchymal tumors [3]. It is also reported that CD99 expression may be associated with the biologic behavior of some tumors because of its roles in T cell apoptosis [3].

Metastasis of MFH occurs most in the lung (90\%), followed by bone (8\%) and liver (1\%). Concerning skin metastasis, only 8 cases of MFH with cutaneous metastasis were previously reported [4], and no cases of in-transit metastasis of MFH have been reported in the English literature so far. Here, we describe a case of MFH with in-transit metastasis. Similar to previous reports [5], this case suggests the importance of an accurate diagnosis for the prediction of prognosis.

\section{Case Report}

A 30-year-old man in good health presented to our department with a 3-year history of an asymptomatic, enlarging tumor on his scalp. The tumor was excised by a private physician 8 years before, but had recurred. Physical examination revealed a skin-colored, elastic hard, well-demarcated tumor on the left side of his occipital region (fig. 1). The tumor size was $40 \mathrm{~mm}$ in diameter. Moreover, there was an elastic hard, satellite nodule located $4 \mathrm{~cm}$ from the tumor. Biopsy specimens taken from the tumor revealed a significant number of minimal atypical spindle or stellate cells in the middle to deep dermis (fig. 2a). Immunohistochemistry showed that a significant number of factor XIIIa-positive cells was disseminated in the tumors (fig. 2b), and CD34-positive cells were limited (fig. 2c). The findings of the laboratory evaluation, including complete blood count, liver function tests and urinalysis, were all within normal limits. We excised the tumor with a 5-mm surgical margin and below the muscle fascia. Histopathology revealed that the main bulk of the tumor was located from the dermis to the subcutaneous tissue ( $\underline{\text { fig. }}$. $\mathrm{a}$ ). The dermal lesion consisted of minimal atypical spindle or stellate cells and foam cells within myxoid matrices (fig. 3b, c). Moreover, CD99 was limited in both the primary and metastatic tumor (fig. 3d). On the other hand, the satellite nodule was composed of densely proliferating spindle cells with moderate pleomorphism arranged in a haphazard fashion (fig. 3e), and, in contrast to the primary tumor, the satellite lesion was located in the subcutaneous tissue (fig. $3 \mathrm{f}$ ). Immunohistochemically, both the primary tumor and the satellite nodule were positive for vimentin and CD31+, and negative for SMA, S100, desmin, HMB-45, Melan-A, AE1/AE3, and Cam5.2. From these results, we made the diagnosis of $\mathrm{MFH}$ with in-transit metastasis in our patient. We examined for possible distal metastasis by computed tomography scan, and detected no metastasis. Five years after the surgical treatment, there was no evidence of local recurrence or metastasis.

\section{Discussion}

We present a case of MFH with in-transit metastasis. In the present case, the primary tumor was mainly located in the dermis, similar to the cutaneous form of $\mathrm{MFH}$, also known as AFX. As the metastasis of MFH occurs mainly through tumor vessels, in-transit metastasis of MFH is extremely rare.

It is difficult to ascertain the biological behavior of MFH in the skin. In general, the prognosis of MFH is suggested to depend on the size of the initial lesion, as well as the depth of the tumor and the histological grade. Superficial tumors of less than $5 \mathrm{~cm}$ in diameter have an excellent prognosis. On the other hand, high-grade lesions situated deep in the skeletal muscle and measuring more than $10 \mathrm{~cm}$ in size are at great risk of metastasis [6]. In our case, the primary tumor was mainly confined to the dermis, had not penetrated into the muscle fascia and was less than $5 \mathrm{~cm}$ in diameter; however, local recurrence with in-transit metastasis occurred 8 years after the primary resection of the tumor. Because recurrence is frequently due to misdiagnosis of the proliferation as 
benign, our case strongly suggests the importance of a precise diagnosis and a complete resection of the primary tumor in the initial phase.

Recently, Hartel et al. [2] reported a higher proportion of CD99 positivity in AFX compared with MFH (94 vs. 15\%) and that the degree of CD99 expression is in part related to their different biologic behaviors. Since CD99 has been shown to play a role in T cell apoptosis [3], they concluded that the strong CD99 expression in AFX may be pathogenetically associated to some degree with a more effective apoptosis of tumor cells and, therefore, correlate with a benign clinical course. In our case, only a slight expression of CD99 was observed both in primary and in-transit metastatic lesions. In other words, the lack of CD99 expression in our case may have been the cause of the in-transit metastasis of the primary tumor.

Regarding treatment, initial wide excision is recommended because of the tendency for local recurrences, which generally increases the risk of metastasis. In the present case, no recurrence occurred over a period of 5 years; however, there was in-transit metastasis together with a history of local recurrence.

\section{Disclosure Statement}

There authors have no financial interests to disclose.

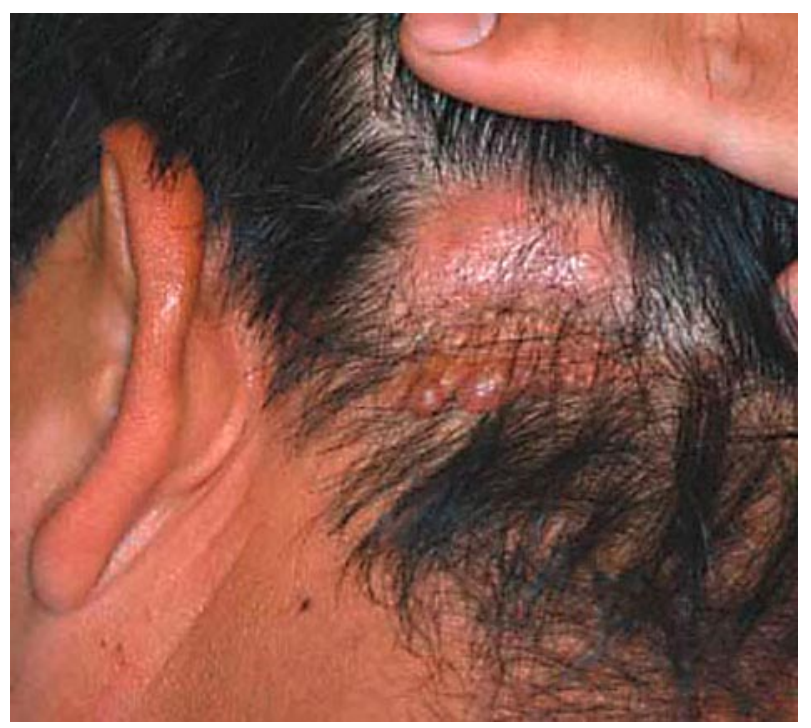

Fig. 1. A skin-colored, well-demarcated tumor located on the left side of the occipital region. 

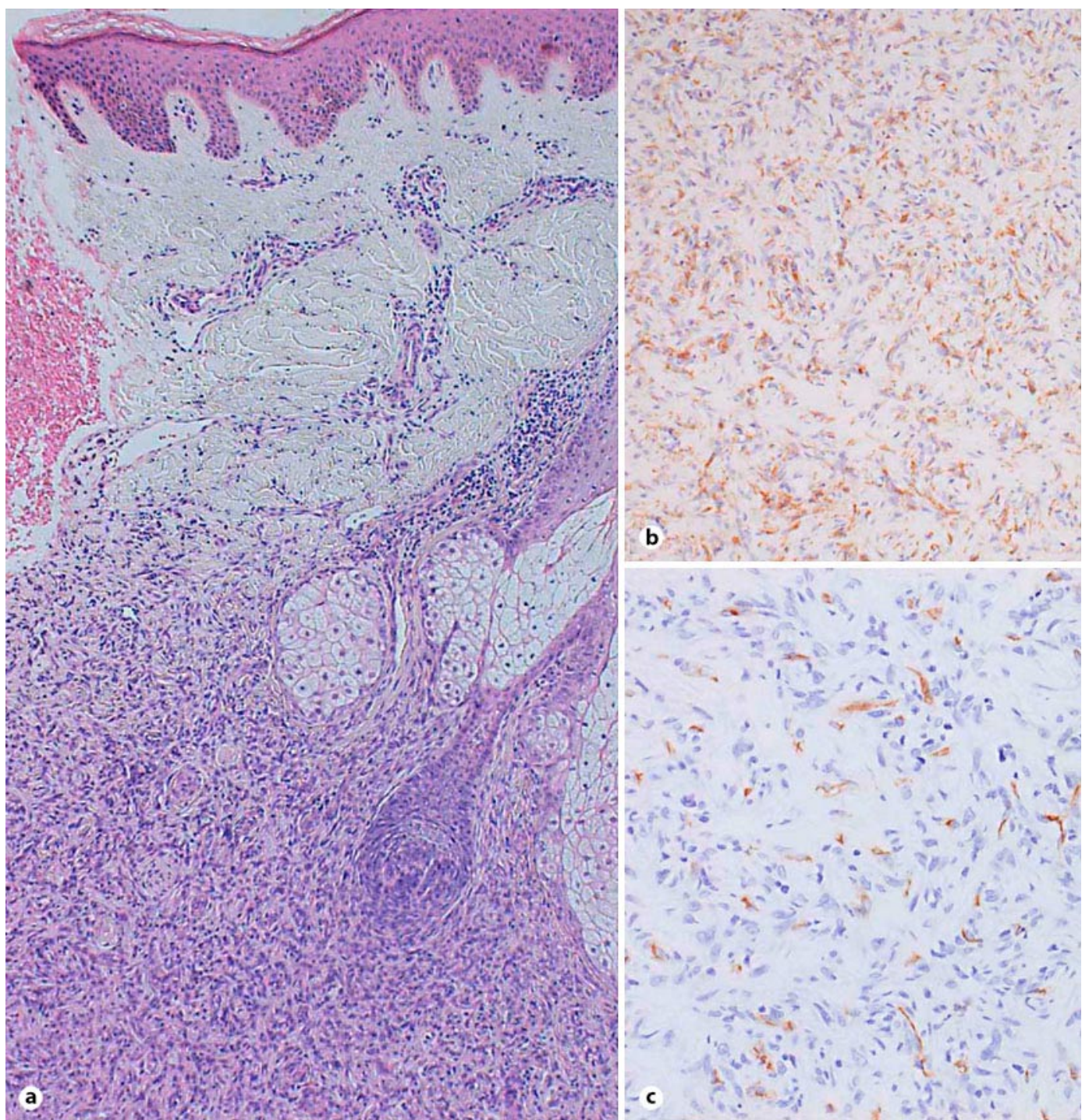

Fig. 2. The biopsy specimen revealed a significant number of minimal atypical spindle or stellate cells in the middle to deep dermis (a). Immunohistochemical staining of the biopsy specimens revealed that the tumor was mainly factor XIIIa positive (b), and CD34 negative (c). a: HE stain, $\times 10$; b: factor XIIIa, $\times 200$; c: CD34 stain, $\times 200$. 


\begin{tabular}{r|l|l|l}
$\begin{array}{c}\text { Case Reports in } \\
\text { Dermatology }\end{array}$ & $\begin{array}{l}\text { Case Rep Dermatol 2011;3:164-169 } \\
\text { DOI: } 10.1159 / 000331324\end{array}$ & August 17, 2011 & $\begin{array}{l}\text { I 2011 S. Karger AG, Basel } \\
\text { ISSN 1662-6567 } \\
\text { www.karger.com/cde }\end{array}$ \\
\hline
\end{tabular}
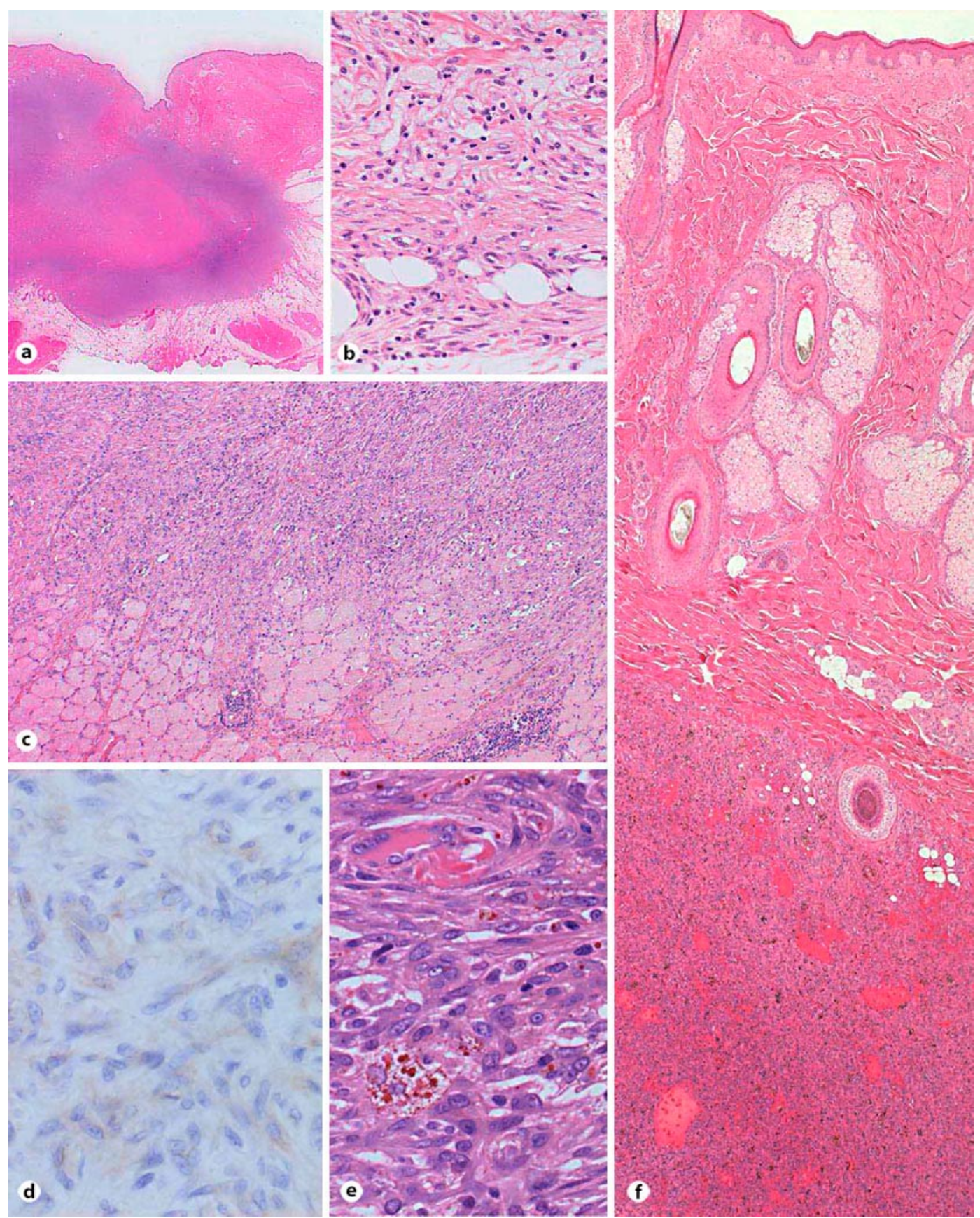

Fig. 3. The main bulk of the primary tumor was located from the dermis to the subcutaneous tissue (a). In the primary tumor, the dermal lesion consisted of scattered, minimal atypical spindle or stellate cells and foam cells with myxoid matrices $(\mathbf{b}, \mathbf{c})$. Immunohistochemical staining of the primary tumor revealed that the tumor was only slightly CD99 positive (d). In-transit metastasis: the tumor was composed of densely proliferating spindle cells with moderate pleomorphism arranged in a haphazard fashion (e). In-transit metastasis: the main tumor was located in the subcutaneous tissue, though, the overlying dermis showed no signs of the tumor $(\mathbf{f})$. HE stain. $\mathbf{a}: \times 5 ; \mathbf{b}: \times 200 ; \mathbf{c}: \times 100$; d: CD99 stain. $\times 400$; e: $\times 400$; $\mathbf{f} \times 50$; original magnification. 


\section{References}

1 Weiss SW, Goldblum JR (eds): Enzinger and Weiss's Soft tissue tumor: fourth edition. St. Louis: Mosby, 2001, p 535.

2 Hartel PH, Jackson J, Ducatman BS, Zhang P: CD99 immunoreactivity in atypical fibroxanthoma and pleomorphic malignant fibrous histiocytoma: a useful diagnostic marker. J Cutan Pathol 2006;33(suppl 2):2428.

-3 Jung KC, Kim NH, Park WS, Park SH, Bae Y: The CD99 signal enhances FAS-mediated apoptosis in the human leukemic cell lines, Jurkat. FEBS Lett 2003;554:478-484.

-4 Lew W, Lim HS, Kim YC: Cutaneous metastatic malignant fibrous histiocytoma. J Am Acad Dermatol 2003;48:S39-S40.

-5 Fujimura T, Okuyama R, Terui T, Okuno K, Masu A, Masu T, Chiba S, Kunii T, Tagami H, Aiba S: Myxofibrosarcoma (myxoid malignant fibrous histiocytoma) showing cutaneous presentation: report of two cases. J Cutan Pathol 2005;32:512-515.

6 Mansoor A, White CR Jr: Myxofibrosarcoma presenting in the skin: clinicopathological feature and differential diagnosis with cutaneous myxoid neoplasm. Am J Dermatopathol 2003;25:281-286. 